\title{
RESULTADOS PRELIMINARES DE LA CRIANZA DE Saguinus mystax (PRIMATES: CALLITRICHIDAE) EN UN GALPON DE REPRODUCCION AL AIRE LIBRE.
}

Sicchar Luis $\left(^{*}\right)$

Tapia Julio $\left({ }^{* *}\right)$

Encamación Filomena $\left(^{* * *}\right)$

\section{RESUMEN}

Desde noviembre de 1981 a diciembre de 1985, se realizó un estudio. Relacionado a la crianza y reproducción de una pareja de Saguinus mystax en asociación con Saguinus fuscicollis, en un galpón de reproducción al aire libre "GRAL", del Centro de Reproducción y Conservación de Primates no Humanos-Iquitos, "CRCP", adecuado a condiciones similares al de su medio ambiente natural. La dieta consistió de frutos regionales, suplementada con una ración de papilla a base de productos lácteos e insectos y pequeños reptiles.

El agua fue proporcionada diariamente en frascos de vidrio; se registraron, asimismo, datos de temperatura, variación ambiental y observaciones sobre la condición de salud de los primates. Complementariamente se realizaron los controles de peso, exámenes de salud y toma de muestras fecales. Durante el estudio, ambas especies tuvieron tres acontecimientos reproductivos, con un $100 \%$ de de nacimientos vivos. $L a$ mortalidad de las crías sucedió unicamente en S. mystax en $50 \%$, en períodos próximos al destete y durante la lactación. Al término del estudio, la población estuvo conformada por la pareja de $\mathrm{S}$. mystax con tres descendientes y la pareja de $\mathrm{S}$. fuscicollis con seis descendientes, todos en óptimas condiciones de salud.

\section{INTRODUCCION}

Saguinus mystax en su ambiente natural, es ubicado normalmente en asociación con Saguinus Fuscicollis (Castro y Soini, 1977). Desde que se inició en 1976 la crianza de esta especie en cautiverio en el Centro de Reproducción y Conservación de Primates no Humanos ( CRCP) del Instituto V eterinario de Investigaciones Tropicales

P.C.: Saguinus mystax, Saguinus fuscicollis- crianza asociada; galpón de reproducción al aire libre y de Altura (IVITA), Iquitos, ha presentado numerosas dificultades. Las parejas reproductoras son acondicionadas en ambientes pequeños, que les ocasionan estrés permanente, a diferencia de un galpón de reproducción al aire

* Director Ejecutivo de Conservación y M anejo (IIA P)

** Proyecto Peruano de Primatología, IVITA - UNM SM

Proyecto Peruano de Primatologla, IVITA - UN M SM 
libre 'GRAL' que, a parte de proporcionarles condiciones de vida similares a los de su ambiente natural, facilitó el acercamiento del observador a los animales. Existe escasa información sobre el comportamiento de S. mystax en cautiverio (Box y M orris, 1980 y Kaumanns, 1982), así como de su crianza (Ogden 1983). Las experiencias de Castro (1980), Málaga (1980-1986) y Villavicencio et al (1986), enfatizan esta deficiencia en los intentos de reproducción en cautiverio. Los resultados que se presenta en este reporte corresponden a la crianza de una pareja de S.mystax que fue acondicionada y asociada con otra de S. fuscicollis en el "GRAL" del CRCP, con condiciones de vida semejantes al ambiente natural, desde noviembre de 1981 hasta diciembre de 1985.

\section{MATERIALES Y METODOS}

\section{Galpón de reproducción al aire libre "GRAL "}

Para los efectos del estudio se construyó un ambiente de madera de $13 \times 9 \times 2.5 \mathrm{~m}$., cerrado con malla tipo mosquitero para el ingreso de la luz solar y lluvia. Esta ambiente presenta tres ventanas de malla metálica para el ingreso de insectos, ubicadas dos de ellas en los extremos del lado derecho y una anterior. El ambiente está rodeado por fuera de matorrales, arbustos y árboles, excepto el lado lateral izquierdo, protegido por un cerco de hojas de palma. Interiormente presenta: a) Suelo con vegetación arbustiva. b) Dos jaulas de malla metálica de $\mid x 1 \times 0.8 \mathrm{~m}$. en la parte media de los lados derecho e izquierdo, usadas para la retención de los animales durante el proceso de acostumbramiento, con una caja de madera laminada como nidal, un comedero en su interior y un bebedero de vidrio exterior y c) Un sistema de perchas paralelas diagonales y verticales, para determinar el uso del espacio vertical y horizontal (Fig 1).

\section{Los animales}

Para el inicio de la investigación se introdujo en el 'GRAL' en noviembre de 1981 una pareja adulta de S. mystax y otra de S. fuscicollis procedentes del estado silvestre. EI primer macho de S. mystax fugó en enero de 1982 y fue reemplazado por otro en abril del mismo año; este último, por no presentar actividad reproductiva, fue reemplazado por un tercero en abril de 1983, permaneciendo hasta el término del estudio. Los dos últimos machos estuvieron previamente en la colonia reproductiva del CRCP, aproximadamente cuatro años. La primera pareja de S. fuscicollis murió en abril de 1982, siendo reemplazada por otra en el mismo mes; estos permanecieron hasta el termino del estudio. 
3. Dieta. -Estuvo constituida de frutos regionales, suplementada con una ración de papilla a base de productos lácteos ( $15 \mathrm{gr}$. Cerelac $\left({ }^{*}\right)$ y $05 \mathrm{gr}$. Sustagen $\left({ }^{* *}\right)$ por animal), suministrados dos veces por día, y agua proporcionada en frascos de vidrios.

Los frutos, como "uvilla" Pourouma cecropiaefolia "guaba" Inga "shimbillo" Inga sp. "guabilla" Inga sp. plátano M usa sp. "papaya" Carica papaya chanchuelo" Rheedia floribunda, "caimito" Pouteria caimito y "tamamuri" Ogeodeia sp., fueron suministrados de acuerdo a la disponibilidad en los mercados de Iquitos y según las estaciones del año.

\section{Procedimiento.}

El manejo de los animales tuvo dos secuencias: a) La fase de acostumbramiento en las jaulas interiores, por un período mínimo de una semana, y b) U so del espacio integral del galpón ( $M$ ayores detalles ver Kaumanns 19821). Durante el estudio se registraron datos de temperatura ambiental, condiciones climáticas y observaciones sobre las condiciones de salud de los animales. Además, cada seis meses eran sometidos a controles de peso y exámenes de salud, incluyendo toma de muestras fecales para análisis parasitológicos.

Cereal para la alimentación infantil producido en el Perú por Nestlé S.A.

** A limento infantil producido por laboratorios M ead Johnson y comercializado en el Peru. 


\section{FIG.1}

5.
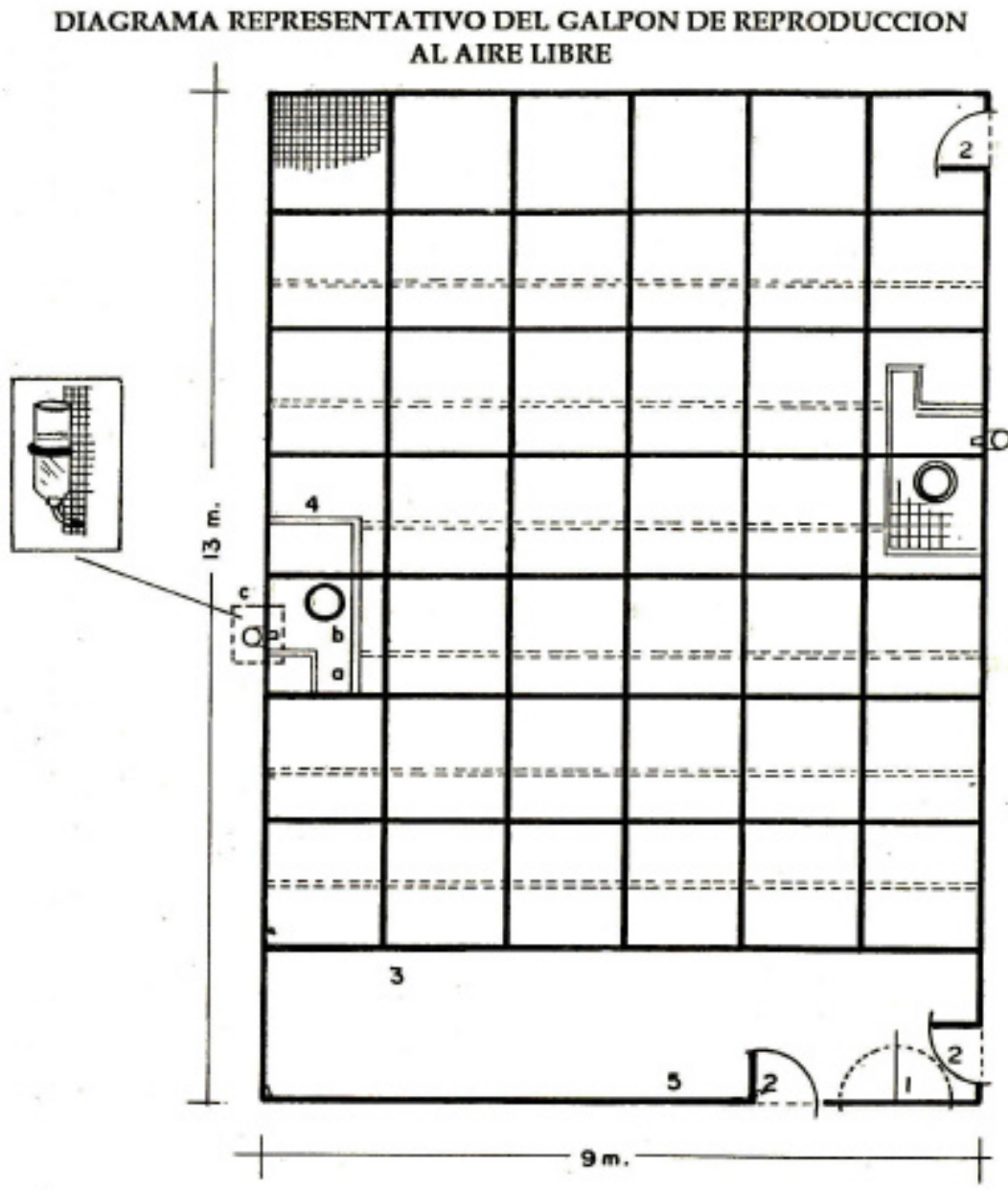

1. Puerta de entrada

2. Ventanas

3. Sistema de perchas

4. Jaulas interiores

4a. Nidal

4b. Comedero

4c. Bebedero

5. Lugar de observación 


\section{$\underline{\text { Resultado }}$}

\subsection{Actividad reproductiva}

Durante el estudio, las parejas de S. mustax y S.fuscicollis tuvieron tres pariciones en diferentes períodos, alcanzando el $100 \%$ de nacimientos vivos (Cuadro $\mathrm{N} \cong 1$ )

La actividad reproductiva del tercer macho de $\mathrm{S}$. mystax se evidenció 15 días después de haber sido introducido en el "GRAL", y el resultado fue la primera carnada en agosto de 1983, la segunda en julio de 1984 y la tercera en junio de 1985. El intervalo entre la primera y la segunda carnada fue de 341 días, y entre la segunda y la tercera de 350 días, En S. fusicollis la primer a carnada se produjo a los 270 días de introducida la pareja en el "GRAL". El intervalo entre la primera y la segunda fue de 289 días, y entre la segunda y la tercera de 673 días.

La mortalidad de las crías se registró sólo en $\mathrm{S}$. mystax por factores externos, que representó el $50 \%$ en edad próxima al destete en la primera carnada y durante la lactación en la segunda carnada (Cuadro № 2).

\section{$52 \quad$ Peso y condición de salud}

EI registro de pesos corresponde sólo a los reproductores de $\underline{S}$. mystax notándose incrementos significativos (Cuadro $\mathrm{N} N$ 3). En este cuadro se puede apreciar que la hembra incrementó su peso en $59 \mathrm{gr}$., desde la fecha de su introducción en el "GRAL" hasta abril de 1983, sin tomar en consideración el peso obtenido en los últimos días de gestación, que fue de 690 gr. Los registros para el segundo y tercer macho, muestran un incremento de 108 y74 gr., respectivamente.

Las variaciones de temperatura (mínima 20 ㄷ y máxima 36 C C) y climáticas (sol, Iluvia, frío), no afectaron la salud de los animales, manteniéndose en óptimas condiciones.

Los análisis de heces determinaron la presencia de Prosthenorchis elegans Strongyloides sp., que fueron tratados con Thiabendazol., persistió Prosthenorchis. Que aparentemente no afectó la salud de $\underline{S}$. mystax cuando las condiciones de alimentación y estrés están ausentes.

La población, al término del estudio, estuvo constituida por adultos, subadultos, juveniles e infantes, de acuerdo a las categorías de edad establecida por Soini (1981 -1982), que en el caso de S. mystax estuvo conformada por la pareja reproductora y tres descendientes (1 sub-adulto y2 juveniles) y en $\underline{S}$. fuscicollis por los reproductores y seis descendientes (4 adultos y 2 infantes) (C uadro $\mathrm{N} N 4$ ). 
CUADRO N으 1

REPRODUCCION DE S.mystax Y S. fuscicollis

Saguinus M ystax

\begin{tabular}{|c|l|l|}
\hline Emparejamiento & Pariciones & Crías \\
\hline A br. 1983 & 05 A go.83 & 2M achos \\
& 12 Jul. 84 & 2No sexados \\
& 27 Jun. 85 & 2 Machos \\
\hline
\end{tabular}

Saguinus Fuscicollis

\begin{tabular}{|c|c|c|}
\hline Emparejamiento & Pariciones & Crías \\
\hline A br. 1982 & 14 Ene. 83 & 2 M achos y Hembra \\
& 30 Oct. 84 & 2 N o sexos y hembra \\
& 03 Set. 85 & \\
\hline
\end{tabular}

CUADRO N 02

MORTALIDAD DE CRIAS EN S. mystax

\begin{tabular}{|c|c|c|c|c|c|}
\hline $\mathrm{N}$ acimiento & M uerte & $\begin{array}{l}\text { Edad } \\
\text { Días }\end{array}$ & Causa & $\begin{array}{c}\mathrm{N} \cong \\
\text { M uertos }\end{array}$ & Camada \\
\hline 05 A g0. 83 & 23 Oct. 83 & 79 & $\begin{array}{l}\text { A ccidente (atsc- } \\
\text { Cado entre } \\
\text { perchas). }\end{array}$ & 1 & 1ra \\
\hline 12 J ul. 84 & 26 Jul. 84 & 15 & Desconocida & 2 & $2 d a$ \\
\hline
\end{tabular}


CUADRO N 3

REGISTRO DE PESOS EN $\underline{\text { s. mystax }}$

\begin{tabular}{l|c|c|c}
\hline Animal & Introducción & Registro & $\begin{array}{c}\text { Peso } \\
\text { (gr) }\end{array}$ \\
\hline Hembra & Nov. 1981 & Nov. 81 & 511,0 \\
& & Abr. 82 & 530,0 \\
& & Oct. 82 & 552,0 \\
Segundo & \multirow{2}{*}{ Abr. 1982 } & Abr. 83 & 570,0 \\
Macho & & Abr. 82 & 465,0 \\
& & Oct. 82 & 553,4 \\
Tercer & Abr. 1983 & Abr. 83 & 573,0 \\
Macho & & Abr. 83 & 511,0 \\
& & Set. 83 & 552,0 \\
& & Mar. 84 & 585,0 \\
\hline
\end{tabular}

\section{CUADRO N4}

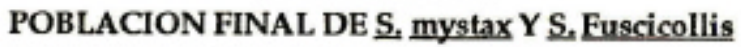

Saguinus mystax

\begin{tabular}{l|c|ccc|c}
\hline \multirow{2}{*}{ Reproductores } & Camada & \multicolumn{3}{|c|}{ Edad de la Progenie } & Total \\
& A & SA & J & I & Animales GRAL \\
\hline 2(HM) & $1 \mathrm{ra}$ & - & $\begin{array}{c}1(514 \\
\text { días })\end{array}$ & - \\
& 3ra & - & - & $\begin{array}{c}2 \\
\text { (18 dias) }\end{array}$ & 5 \\
\hline
\end{tabular}

Saguinus fuscicollis

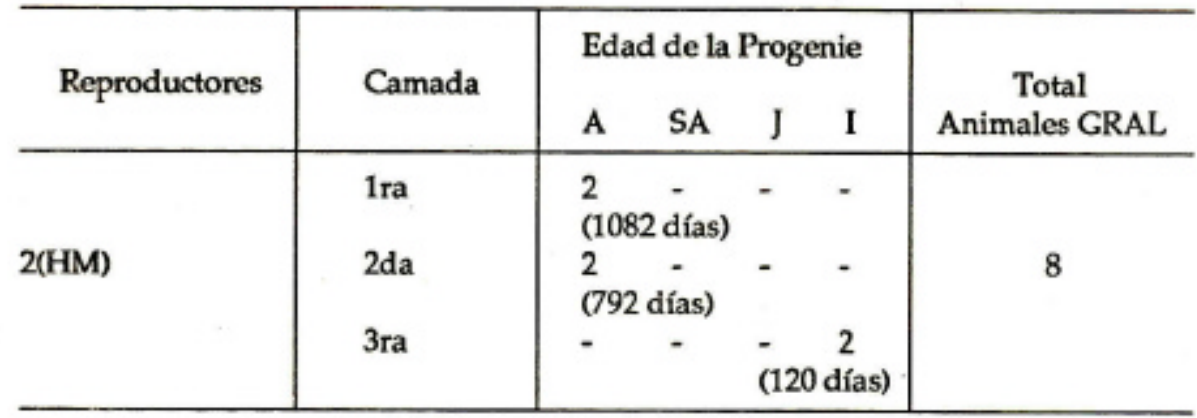




$\begin{array}{lll}\mathrm{HM} & = & \text { Hembra y macho (Pareja reproductora) } \\ \mathrm{A} & = & \text { Adultos } \\ \mathrm{SA} & & \text { Subadultos } \\ \mathrm{J} & = & \text { Juveniles } \\ 1 & = & \text { Infantes }\end{array}$

\section{Discusión}

La biología reproductiva de los callitrícidos es notable en relación a otros primates. Los tamarinos y tities se caracterizan por su rápido potencial de incremento poblacional (Garber, 1984). Sin embargo, este potencial en S. mystax no muestra éxitos en la crianza en cautiverio, limitación que es resaltada por Box and Morris (1980), Málaga (1980 1986) y Villavicencio et al (1986). Este último autor menciona que, en condiciones como la del CRCP S. mystax pierde la estacionalidad de sus pariciones exhibidas en el campo. M álaga (1980), señala que en el CRCP la actividad reproductiva de S. mystax se mantiene por debajo de los niveles normales, siendo uno de los problemas la supervivencia de las crías nacidas. Ogden (1983), reporta la dificultad de criar S. mystax en condiciones de laboratorio y que los índices de producción de crías logradas son inferiores a las de otras especies de callitrícidos, por la incidencia de abortos y partos muertos. Posteriormente, Málaga et al (1986) y Villavicencio et al (1986), reportan la ocurrencia de neumonías, enteritis y prosthenorchiasis. El síndrome consumptivo y la insuficiencia hepática de origen desconocido, son otros de los problemas citados por Málaga (1986) como causa de la dificultad de crianza de S. mystax. A simismo, Villavicencio et al (1986), señala la ocurrencia de un al to porcentaje de abortos en la colonia del CRCP. Sin embargo, durante el estudio no se presentaron estos acontecimientos. A bbot y Hearn (1978), citados por Evans and Poole (1983), reportan para otra especie de callitrícido una alta incidencia de abortos espontaneos en hembras adultas primíparas. Durante el estudio las condiciones de salud y la reproducción de $\mathrm{S}$. mystax fueron óptimas, con pariciones anuales, mostrando buena eficiencia reproductiva. Estas fueron favorecidas por las condiciones de mayor espacio que ofrecíá el "GRAL", suelo con vegetación arbustiva, presencia de insectos, pequeños reptiles, luz solar, Iluvia y corrientes de aire. Estos factores contrastan con los pequeños espacios de las jaulas de reproducción en cautiverio, tanto del CRCP como de otros laboratorios. La eficiencia reproductiva de $\mathrm{S}$. mystax fue anual, mientras que la de $\mathrm{S}$. fuscicollis fue irregular, debido a la presencia de un rango entre pariciones desde 289 hasta 673 días, no obstante haber compartido el ambiente y dieta. Se asume que esta irregularidad fue ocasionada por el desarrollo de la estructura social de S. fuscicollis 0 el estrés provocado durante el manipuleo del S. mystax para el pesaje. 


\section{BIBLIOGRAFIA}

Box, H.O.;J.M. M orris. (1980). Behavioral observations on captive pairs of wild caught tamarins (Sanguinus mystax) Primates 21:53-65.

Castro, R.;Soini, P. (1977). Field studies on Saguinus mystax and other callitrichids in A mazonian Perú; in Kleiman, the biology and conservation of callitrichidae, pp. 73-78 (Srnithsonian Institution Press, W ashington).

Castro, R. (1980): Resultados preliminares sobre las investigaciones de Saguinus mystax (Primates: Callitrichidae), 1976 - 1979. Evaluación de la población en áreas naturales, II Manejo de cautiverio: E.C.R.P. "Informe presentado a la Organización Panamericana de la Salud, Washington, D.C.

Evans, S. and T.B. Poole. 1983. Pair-Bond Formation and Breeding Success in the Common M armosets, Callithrix jacchus. Int. 1. of Primatol. 4:83-97,

Garber, P.A.; L M oya; C. Málaga. 1984. A preliminary fleld study of the moustached tamarin monkey Saguinus mystax) in northeastern Perú: Questiorts concerned with the evolution of a communal breeding system. Folia Primatol. 42:17-32.

Kaumanns, W. 1982. Verhaltensbeobachtungefl an Schnurrbarttamarinen (Saguinus mystax mystax). Zeistschrift des Kölner Zoo. 25: 107- 117.

Málaga, C.1980. “Informe correspondiente al año 1980. A M R0-31 77, Iquitos - Perú.

Málaga, C.;E. Montoya; E. Villavicencio, 1986. Prevalencia de Parasitosis Gastro Intestinales y otras enfermedades en primates No-Humanos del Centro de Reproducción y Conservación de Primates (CRCP). Iquitos - Perú.

Ogden, 1. 1983. Reproduction of Wild-Caught Tamarins (Saguinus mystax mystax) under laboratory conditions. L M ed. Primatologv 12: 343 - 345.

Soini, P. 1981. Ecología y Dinámica Poblacional del Pichico Común Saguinus fuscicollis (Primates: Callitrichidae). Informe Pacaya № 4. Dirección Regional de Agricultura, Dirección Forestal y de Fauna. Iquitos - Perú.

Soini, P. 1982. Distribución Geográfica y Ecología Poblacional de Saguinus mystax (Primates: Callitrichidae). Informe Pacaya № 6, ORDEL ORETO, Dirección Regional de A gricultura, Dirección Forestal y de Fauna. Iquitos - Perú. 
Villavicencio, E.; E. M ontoya; C. Málaga. 1986. A spectos reproductivos y sanitarios de la colonia de Saguinus mystax del Centro de Reproducción y Conservación de Primates. Iquitos - Perú. 